DOI 10.37882/2500-3682.2020.10.04

\title{
КЛИНИЧЕСКИЕ И ПСИХОЛОГИЧЕСКИЕ МОДЕЛИ ПАТОЛОГИЧЕСКИХ КОЖНЫХ ОЩУЩЕНИЙ
}

\section{CLINICAL AND PSYCHOLOGICAL MODELS OF PATHOLOGICAL SKIN SENSATIONS \\ A. Ermusheva}

Summary: The complexity of empirical study of psychological mechanisms of pathological bodily sensations is associated with attention to particular aspects of this pathology in different approaches: clinical-descriptive, psychoanalytic, cognitive-behavioral, and psychology of corporeality. The interest of clinicians in the subjective nature of patients' complaints and the need to find a link between various psychological aspects form the topicality of continuing to research the problem. The study of mediation of pathological skin sensations in psychodermatological disorders seems promising, as it makes possible to take into account the ideas on the individualisation and the meaning of bodily experience in psychology of corporeality, the role of insufficient ability to effectively process painful experience using symbolic and language means in psychoanalysis and the participation of cognitive processes in the experience of pathological bodily sensations in cognitive-behavioral approach.

Keywords: pathological bodily sensations, medically unexplained symptoms, psychodermatological disorders, psychology of corporeality.

\author{
Ермушева Анастасия Алексеевна \\ Ассистент, Первый МГМУ им. И.М.Сеченова \\ (Сеченовский Университет) (Москва) \\ aermusheva@gmail.com
}

Аннотация: Сложность эмпирического исследования психологических механизмов патологических телесных ощущений вызвана сфокусированностью на отдельных аспектах этой патологии в различных подходах: клинико-описательном, психоаналитическом, когнитивно-поведенческом и психологии телесности. Интерес клиницистов к субъективной природе жалоб пациентов, а также необходимость соотнесения различных психологических аспектов формирует актуальность продолжения изучения проблемы. Представляется перспективным развитие исследований патологических кожных ощущений при психодерматологических расстройствах в аспекте их опосредования, что позволяет реализовать их изучение с учетом индивидуализированности и означения телесного опыта с позиций психологии телесности, роли недостаточной способности к эффективной переработке болезненных переживаний с помощью символических и языковых средств в рамках психоаналитического подхода и участия когнитивных процессов в переживании патологических телесных ощущений в рамках когнитивно-поведенческого подхода.

Ключевые слова: патологические телесные ощущения, медицински необъяснимые симптомы, психодерматологические расстройства, психология телесности.

Наибольшее внимание в исследованиях патологических кожных ощущений в рамках клинико-описательного подхода уделяется зуду. В исследованиях механизмов психогенного зуда как одного из «медицински необъяснимых дерматологических симптомов» изучаются как биохимические, так и психопатологические аспекты[24]. Активно исследуются нейронные механизмы зуда: общность ощущений зуда и боли, основывающаяся на значительном совпадении между периферическими медиаторами и рецепторами; центральные нейрональные пути, не связанные с ноцицептивными рецепторами, рецепторы, специфические для зуда, и медиаторы, участвующие в восприятии зуда[39]. Значительное внимание уделяется интенсивности этого ощущения, тогда как его другие качества исследованы в меньшей степени[22]. В исследованиях сенсорного и аффективного компонентов зуда показано, что именно аффективный компонент является предиктором психологических осложнений заболевания и снижения качества жизни пациентов[59]. При этом значимость психологической и психиатрической коморбидности подчеркивается в практически ориентированных исследованиях организации помощи пациентам в психодерматологических клиниках[25].

В исследовании телесных ощущений как проявлений дерматозов отдается предпочтение визуально-аналого- 
вым шкалам[58], электрофизиологическим показателям, а также особенностям химически вызванных ощущений у человека и животных[37]. При этом субъективная реальность этих ощущений остается относительно малоизучена. Встречаются исследования того, как пациенты характеризуют зуд и другие телесные ощущения, однако в основном они проводятся при истинных дерматозах например, при атопическом дерматите[22]. Вместе с тем, отмечается нарастание интереса к восприятию и субъективным оценкам кожных ощущений, поскольку происходит накопление данных об их расхождении с объективными показателями даже при истинных дерматозах[40], что также открывает перспективы изучения патологических телесных ощущений.

В рамках клинико-описательного подхода отмечается особая роль патологических кожных ощущений в становлении психических расстройств, реализующихся в пространстве кожного покрова[11], или психодерматологических расстройств, характеризующихся типичным выявлением самоиндуцированных и соматоформных кожных нарушений, напоминающих клинические проявления дерматологической патологии при отсутствии объективных проявлений какого-либо известного дерматоза[26]. К этим расстройствам относятся дерматозойный бред, синдром ограниченной ипохондрии, синдромы импульсивных и компульсивных экскориаций.

При дерматозойном бреде выявляются идеи заражения кожными паразитами или какими-либо живыми или неживыми патогенами, несмотря на их отсутствие по результатам объективных исследований[44], они ассоциированы с тактильными галлюцинациями, дополняемыми зрительными (иногда слуховыми и обонятельными) иллюзиями или галлюцинациями[11]. В диагностических критериях описывается разнообразие патологических кожных ощущений, включающих зуд, ползнание, пощипывание и покалывание[30, 38]. У пациентов имеются значительные повреждения кожных покровов как результат их борьбы с мнимыми паразитами[32, 34]. В аспекте патогенеза обсуждаются нарушения интероцепции, неправильные обработка и интерпретация воспринимаемых ощущений как способствующие формированию галлюцинаций[35].

При синдроме ограниченной ипохондрии коэнестезиопатии включают зуд по типу интрадермальной дизестезии, идиопатические алгии, тактильные иллюзии и телесные фантазии, ассоциированные со сверхценными образованиями с картиной ипохондрической одержимости[54]. Фрагменты кожи и подкожной клетчатки, извлекаемые пациентами из соответствующих ощущениям областей, воспринимаются как чужеродные объекты и патологические, не свойственные живым тканям образования. При этом пациенты испытывают определенные, отчетливые и неизменные патологические теле- сные ощущения лишь в ограниченной области[21].

При синдромах импульсивных и компульсивных экскориаций пациенты сообщают о навязчивых и непреодолимых импульсах к расчесыванию[29] с ощущениями зуда по типу интрадермальной или эпидермальной дизестезии[11]. Расстройства характеризуются различными манипуляциями (расчесыванием, сдиранием, сдавливанием), производимыми с кожей[34]. Физические следы экскориаций располагаются в легкодоступных для пациентов местах - лицо, руки, верхняя часть спины[60]. Клинические исследования указывают как на компульсивную, так и импульсивную природу заболевания[46]. По результатам исследования особенностей перцепции выявляется склонность пациентов к избеганию ощущений в сочетании с высокой сенсорной чувствительностью[28].

Таким образом, в исследованиях клинико-описательного подхода фиксируется интерес к субъективной природе жалоб пациентов, специфике и распространенности патологических кожных ощущений, а также их роли и значении в клинической картине психодерматологических расстройств, что однако нуждается в дополнениях со стороны психологических исследований вследствие многообразия и разнородности факторов, обуславливающих медицински необъяснимые симптомы.

Началу обсуждения механизмов патологических кожных ощущений в рамках психоаналитического подхода послужило введение 3. Фрейдом[17] механизма конверсии, по которому в качестве символического выражения желания возникает истерический симптом, способный проявляться как психосенсорное нарушение. Однако для выделения нюансов дискуссии вокруг патологических кожных ощущений следует отметить, что в психоаналитических работах обсуждаются особая связь психики с кожей и ее роль в становлении самосознания и развития психической жизни. Понятие «Я-кожа» отражает, как на ранних этапах развития Я ищет опоры и поддержки в коже тела, что представляет как бы гомологичность функций Я и телесной оболочки ограничивать, контейнировать и организовывать[1]. Развитие представлений о себе базируется на ранних ощущениях с поверхности тела, кожа обеспечивает раннее взаимодействие между внутренним и внешним[52]. Кожа рассматривается как средство коммуникации между матерью и младенцем, будучи посредником для физического контакта, успокоения удерживающего и удерживаемого, а также для передачи запаха, прикосновения, вкуса и тепла - ощущений, способных быть источником удовольствия и близости[8]. Также кожа - орган-мишень для фантазий и страхов, что может использоваться в разворачивании психопатологических симптомов, и тогда кожная поверхность становится «местом аварийной разрядки»[16]. В зрелом возрасте при невозможности 
пережить и прочувствовать психологически значимые события могут актуализироваться патологические ощущения, являющиеся возвратом к более ранним способам реагирования с помощью «примитивных психических сообщений»[5].

Основным направлением работ психоаналитиков в области психодерматологии становится установление связи между кожными ощущениями и вытесненными желаниями[2]. Зуд рассматривается как конверсионный симптом, «специфический зуд у специфической личности»[2, с. 44]. У пациентов с кожными проблемами, ассоциированными с психическими расстройствами, обсуждаются трудности формирования зрелой идентичности, патология привязанности, недостаточная сформированность различий между внутренним и внешним и слабость возможностей обработки информации[55].

При обобщении данных литературы отмечается сохраняющаяся актуальность представлений о роли в образовании патологических кожных ощущений недостаточной способности взрослого человека к эффективной переработке болезненных для него переживаний с помощью символических и языковых средств. Однако следует отметить, что ретроспективная природа некоторых измерений в современных исследованиях, выполненных в парадигме психодинамического подхода, подвергается критике и признается уязвимой в отношении ошибок припоминания и малой численности выборок[19].

В рамках когнитивно-поведенческого подхода основное внимание исследователей сосредоточено на идеаторной и поведенческой составляющих психических расстройств, сопровождающихся жалобами на патологические телесные ощущения. Патологические телесные ощущения расцениваются как один из факторов формирования предположений о наличии заболевания при ипохондрии[50]. На модели соматоформных и ипохондрических расстройств описаны когнитивные механизмы, способствующие повышению тревоги о здоровье и фиксации патологических телесных ощущений: соматосенсорная амплификация, атрибутивный стиль, особенности точности и чувствительности телесного восприятия. В отношении соматосенсорной амплификации приводятся критические замечания о невозможности выделения ее как независимого фактора и единственного объяснительного механизма, обсуждаются корреляции феномена со степенью выраженности тревоги, депрессии, негативной аффективности и нейротизма[33]. В исследованиях атрибуции соматических ощущений был выделен особый органо-сенситивный атрибутивный стиль, связывающий соматические симптомы в большой степени с тяжелыми заболеваниями и недооценивающий или игнорирующий естественные и нормальные причины[45]. В отношении роли интра- цептивной точности и чувствительности предполагается, что жалобы на патологические телесные ощущения могут быть следствием меньшей точности интрацептивного восприятия или необъективной интрацептивной чувствительности[51].

Несмотря на значительный интерес к факторам патологических телесных ощущений, немногие представления о перечисленных механизмах рассматривались применительно к патологическим кожным ощущениям. В дерматологической клинике в качестве факторов, усиливающих патологические кожные ощущения, рассматриваются такие психологические особенности, как преобладание эмоций негативного характера, повышение уровня тревоги и предрасположенность к неадаптивным стратегиям совладания со средовыми воздействиями и самой ситуацией болезни[57]. У пациентов с патологическими кожными ощущениями как правило, отмечается снижение толерантности к телесным сенсациям вообще и к зуду, в частности. Выявляются изменения в процессах обработки информации: чрезмерно повышенное внимание к кожным ощущениям, интерпретационные ошибки и склонность к их катастрофизации. Предрасположенность к негативным интерпретациям патологических кожных ощущений способствует повышению интенсивности зуда и формированию установки на беспомощность. Большее внимание к возникновению ощущений значимо связано с более высокими показателями интенсивности переживаний зуда[56]. В отношении психогенного зуда рассматривается возможность исследования действия механизма соматосенсорной амплификации[43].

Наряду с идеаторной составляющей, значительное внимание уделяется поведению пациентов с психодерматологическими расстройствами, сопровождающимися жалобами на патологические кожные ощущения. Зачастую используется поведенческое определение зуда как сенсации, которая вызывает стремление к расчесыванию[23]. Расчесывание же определяется как универсальный, неосознаваемый и автономный ответ на патологические кожные ощущения[57]. Этот ответ вызывает временное облегчение, что закрепляет поведенческую реакцию и способствует формированию патологического цикла «зуд-расчесывание-зуд»[39]. Закрепление такой поведенческой реакции в крайних формах может приводить к появлению расчесов в ответ на любой стрессор, не обязательно ощущение зуда[57].

Для психотерапевтического воздействия на патологические кожные ощущения и сопровождающие их действия разрабатываются когнитивно-поведенческие методы, направленные, прежде всего, на изменения привычек пациентов $[29,53]$. При этом следует отметить, что в теоретических моделях и практических рекомендациях мало внимания уделяется особенностям кожных 
ощущений пациентов, скорее побуждениям к расчесыванию.

Таким образом, в рамках когнитивно-поведенческого подхода накоплены данные о разнообразных когнитивных и поведенческих аспектах психических расстройств, сопровождающихся жалобами на патологические телесные ощущения. Однако в литературе отмечается необходимость соотнесения когнитивных механизмов и поведенческих аспектов друг с другом и невозможность сведения наблюдаемых психологических особенностей к какому-либо единичному фактору.

В отечественной психологии телесности с опорой на положения культурно-исторической концепции Л.С. Выготского телесность понимается как следствие смыслового, эмоционально-ценностного отношения, понятийного определения и объяснения, действенно-практического обращения с телесными явлениями[7], в том числе телесными ощущениями. Телесные впечатления формируются в результате активного восприятия и организации интрацептивных ощущений с участием процессов опосредования. К этим процессам относится категоризация телесных ощущений, в результате которой ощущения из зыбких и неопределенных оформляются в более конкретные, получают свою локализацию, интенсивность, модальность, соотносятся с культурными, перцептивными и языковыми эталонами[12]. Так, переживания телесных ощущений зависят от преобладающих в культуре медицинских воззрений, в частности от доминирующего в настоящем времени «ипохондрического» дискурса, обслуживаемого средствами массовой информации[4]. Модификация и порождение необходимых для подтверждения мифа болезни ощущений происходят через выбор и усвоение соответствующих означаемых, в зависимости от предположений и ожиданий человека, меняется и актуальный набор категорий[14].

Субъективное влияние на телесные ощущения проявляется в отношении к телесным явлениям в структуре потребностей, мотивов и ценностной ориентации человека. Смысловое опосредование может проявляться в том, что интрацептивные ощущения и телесные функции становятся средствами выражения не собственных природных потребностей, а других, не связанных с ними напрямую. Достижение состояния здоровья, проявляющееся в соответствующих телесных ощущениях, связывается с потребностью в самореализации у пациентов с расстройствами личности, в клинической картине которых присутствует развернутый ипохондрический синдром[3]. Личностный смысл рассматривается как дополнительный фактор риска формирования телесных ощущений при выполнении задачи на саморегуляцию в ситуации истинной и ложной биообратной связи[6].

Исследования особенностей когнитивной перера- ботки и опосредования феноменов телесности предоставляют сведения о нарушениях при различных формах патологии - как соматических, так и психических расстройствах. С помощью семантических методов были выявлены особенности словарей интрацептивныхх ощущений при онкологических (рак желудка) и кардиологических (инфаркт миокарда) заболеваниях: в структуре словаря четко выделяется центральный блок категорий, соответствующий ощущениям, обусловленным конкретными, физическими симптомами соматической болезни, вокруг которых группируются все остальные интрацептивные признаки, как физические, так и эмоциональные. Для соматоформных, ипохондрических и шизотипических расстройств, протекающих в отсутствие объективно диагностируемого соматического заболевания, характерно значительное расширение словаря, метафоричность в описаниях, меньшая ясность и плохая локализованность ощущений, характеристика болезни за счет «интрацептивно переживаемых эмоциональных состояний»[12]. При исследовании пациентов с синдромом раздраженного кишечника показано, что тяжесть симптомов заболевания у пациентов была в большой степени связана с нарушением восприятия внутренних телесных ощущений: в описании болезненного состояния в лексиконе пациентов было значительно больше слов, относящихся к языку не телесных ощущений, а к сфере эмоциональных состояний[9]. В качестве предикторов риска соматизации обсуждаются выявленные при соматоформных расстройствах расширение объема интрацептивного словаря обычного самочувствия, увеличение в нем конкретных и психических дескрипторов, чрезмерное использование метафор в описании внутренних телесных ощущений, уменьшение преобладания психических дескрипторов над телесными[10]. Результаты исследований своеобразия нарушений в области телесного восприятия показали, что у пациентов с соматоформными расстройствами на модели зрительно-тактильной и визуально-кинестетической иллюзий выявляются ригидность и измененность мультисенсорной интеграции[47]. В целом, жалобы на телесные ощущения могут рассматриваться как проявления общего дистресса и соотносятся с жалобами на психическое состояние даже у условно здоровых испытуемых[31].

Перспективность продолжения исследования опосредования телесного опыта при патологических кожных ощущениях иллюстрируется результатами психологического исследования телесного опыта болезни пациентов с психодерматологическими синдромами. Так, были выявлены особенности словаря болезненных ощущений, ассоциированные с психопатологическими характеристиками каждого из психических расстройств, реализующихся в пространстве кожного покрова (дерматозойный бред, синдром ограниченной ипохондрии, синдромы импульсивных и компульсивных экскориаций, соматоформный зуд)[15]. 
Следует дополнить, что проблематика исследований психологических аспектов функционирования кожи в психологии телесности связана с объективацией границ телесности: кожная оболочка - это «место», где заканчивается человеческое тело и начинается окружающая среда[13]. Внешняя и внутренняя границы телесности связаны между собой, и эта связь проявляется в форме адаптационно-регуляторных феноменов, характерных для больных с разной направленностью инвазивного воздействия и степенью тяжести патологии. Так, на примере псориаза и атопического дерматита были выявлены отличительные особенности границ телесного образа «Я»[18].

Таким образом, в отечественной психологии телесности исследуются механизмы патологических телесных ощущений в аспекте их опосредования и означения с учетом сложности типологического описания телесного опыта пациентов с различной патологией - как соматическими, так и психическими расстройствами.
Итак, анализ литературы выявляет сложность взаимодействия и множественность факторов, участвующих в возникновении патологических телесных ощущений. Интерес клиницистов к субъективной природе жалоб пациентов на патологические кожные ощущения, а также необходимость соотнесения различных психологических аспектов этих телесных феноменов формирует актуальность продолжения исследований. Представляется перспективным развитие исследований патологических кожных ощущений при различных психодерматологических расстройствах в аспекте их опосредования, что позволяет реализовать их изучение с учетом индивидуализированности и означения телесного опыта с позиций психологии телесности, роли недостаточной способности к эффективной переработке болезненных переживаний с помощью символических и языковых средств в рамках психоаналитического подхода и участия когнитивных процессов в переживании патологических телесных ощущений в рамках когнитивно-поведенческого подхода.

\section{ЛИТЕРАТУРА}

1. Анзье Д. Я-кожа. Ижевск: $\mathrm{ERGO}, 2011.302$ с.

2. Психология и психопатология кожи: тексты. / Сост. и науч. ред. С.Ф. Сироткин, М.Л. Мельникова. Ижевск: ЕRG0; М.: Когито-Центр, 2011.384 с.

3. Виноградова М.Г., Ермушева А.А., Шабанова А.А. К проблеме исследования познавательной деятельности при расстройствах личности // Сб. мат. V Международной научно-практической конференции «Психология и педагогика в системе гуманитарного знания». М., 2013. С. 36-42.

4. Рассказова Е.И., Гульдан В.В., Тхостов А.Ш. Психологическое содержание «ипохондрического дискурса»: связь ценности заботы о здоровье с отношением к телу, болезни и лечению // Вестник ЮУрГУ. Серия «Психология». 2016. T.9, N 2. С. 60-70.

5. Макдугалл Дж. Театры тела: Психоаналитический подход клечению психосоматических расстройств. М.: «Когито-Центр», 2013. 215 .

6. Рассказова Е.И., Мигунова Ю.М., Азиатская Г.А. Чувствительность к обратной связи и соматизация: провокация телесных ощущений при истинной и ложной биологической обратной связи // Теоретическая и экспериментальная психология. 2018. Т. 11, N 1. С. 18-27.

7. Николаева В.В., Арина Г.А. Клиническая психология телесности: принципы культурно-исторического анализа предмета // Психосоматика: телесность и культура. /Под ред. В.В. Николаевой. М.: Академический Проект, 2009. С. 49-72.

8. Пайнз Д. Бессознательное использование своего тела женщиной. СПб.: Б.С.К.: Вост.-Европ. ин-т психоанализа, 1997. 193с.

9. Полуэктова Е.А., Курбатова А.А., Рупчев Г.Е., Шептулин А.А., Ивашкин В.Т. Роль эмоциональных расстройств, личностных особенностей и нарушения интрацептивных ощущений в формировании соматических симптомов у больных с синдромом раздраженного кишечника // Российский журнал гастроэнтерологии, гепатологии, колопроктологии. 2013. Т.23, N 6. С. 20-28.

10. Рупчев Г.Е. Психологическая структура внутреннего телесного опыта при соматизации (на модели соматоформных расстройств): дис ... канд. психол. наук. М., 2000. $128 \mathrm{c}$.

11. Смулевич А.Б., Романов Д.В., Львов А.Н. Дерматозойный бред и ассоциированные расстройства. М.: ГЭОТАР-Медиа, 2015. 208с.

12. Тхостов А.Ш. Психология телесности. М.: Смысл, 2002. 287с.

13. Тхостов А.Ш., Бескова Д.А. Телесность как пространственная структура // Психология телесности между душой и телом. / Ред.-сост. В.П. Зинченко, Т.С. Леви. М.: АСТ: АСТ МОСКВА, 2005. С. 236-252.

14. Тхостов А.Ш., Елшанский С.П., Рупчев Г.Е. Объем и категориальная структура словаря внутренних ощущений при разных заболеваниях // Ежегодник Российского психологического общества. 2003. Т. 7. С. 563-565.

15. Тхостов А.Ш., Виноградова М.Г., Ермушева А.А., Романов Д.В. Психологические аспекты патологических телесных ощущений при психических расстройствах, реализующихся в пространстве кожного покрова // Психические расстройства в общей медицине. 2014. N 1. С. 14-25.

16. Фенихель 0. Психоаналитическая теория неврозов. М.: Академический проект, 2004. 848 с.

17. Фрейд 3., Брейер Й. Исследования истерии // Фрейд 3. Собрание сочинений в 26 томах. Том 1. СПб: Вост.-Европ. ин-т психоанализа, 2005. 454с.

18. Штрахова А.В., Харисова А.Р. Телесный образ «Я» и его границы у больных с атипичными дерматитами. Сообщение 2 // Вестник ЮУрГУ. Серия «Психология». 2014. T.7, N 1. С. 102-112

19. Besiroglu L., Akdeniz N., Agargun M.Y., Calka 0., Ozdemir 0., Bilgili G.S. Childhood traumatic experiences, dissociation and thought suppression in patients with 'psychosomatic' skin diseases // Stress and Health. 2009. N 25. p. 121-125. 
20. Cassal C., Ferrão Y. Psychiatric Diseases // Dermatology in Public Health Environments: A Comprehensive Textbook. Eds. Rangel Bonamigo R., Dornelles S.I.T. Springer International Publishing Switzerland, 2018. p. 1035-1055.

21. Dai W., Yu S., Liang J., Zhang M. Nummular headache: Peripheral or central? One case with reappearance of nummular headache after focal scalp was removed, and literature review // Cephalalgia. 2013. N 33(6). p. 390-397.

22. Dawn A., Papoiu A.D.P., Chan Y.H., Rapp S.R., Rassette N., Yosipovitch G. Itch characteristics in atopic dermatitis: results of a web-based questionnaire // British Journal of Dermatology. 2009. N 160(3). p. 642-644.

23. Feramisco J.D., Berger T.G., Steinhoff M. Innovative Management of Pruritus // Dermatologic Clinics. 2010. N 28. p. 467-478.

24. Ferreira B.R., Pio-Abreu J.L., Reis J.P., Figueiredo A. Medically unexplained dermatologic symptoms and psychodermatology // Journal of the European Academy of Dermatology and Venereology. 2018. N 32(12). p. e447-e448.

25. Goyal N., Shenoi S., Prabhu S.S., Sreejayan K., Munoli R., Rai S. Psychodermatology liaison clinic in India: a working model // Tropical Doctor. 2018. N 48(1). p. 7-11.

26. Gupta M.A., Gupta A.K. Self-induced dermatoses: a great imitator // Clinics in Dermatology. 2019. N 37(3). p. 268-277.

27. Haller H., Cramer, H., Lauche, R., Dobos, G. Somatoform disorders and medically unexplained symptoms in primary care: a systematic review and metaanalysis of prevalence // Deutsches Ärzteblatt International. 2015. N 112. p. 279-287.

28. Houghton D.C., Alexander J.R., Bauer C.C., Woods D.W. Abnormal perceptual sensitivity in body-focused repetitive behaviors // Comprehensive Psychiatry. 2018. N 82. p. $45-52$.

29. Jafferany M., Patel A. Skin-picking disorder: a guide to diagnosis and management // CNS Drugs. 2019. N 33(4). p. 337-346.

30. Katsoulis K., Rutledge K.J., Jafferany M. Delusional infestation: a prototype of psychodermatological disease // International Journal of Dermatology. 2020. N 59(5). p. 551-560.

31. Khrushchev, S., Rasskazova, E., Tkhostov, A., and Guldan, V. Somatic and mental complaints in the normative population: General "ill-being" or somatic "mask" of mental problems? // European Psychiatry. 2018. N 48(S1). p.259.

32. Koblenzer C.S. Cutaneous manifestations of psychiatric disease that commonly present to the dermatologist - diagnosis and treatment // The International Journal of Psychiatry in Medicine. 1992. N 22. p. 47-63.

33. Krautwurst S., Gerlach A.L., Gomille L., Hiller W. Health anxiety - An indicator of higher interoceptive sensitivity? // Journal of Behavior Therapy and Experimental Psychiatry. 2014. N 45. p. 303-309.

34. Krooks J.A., Weatherall A.G., Holland P.J. Review of epidemiology, clinical presentation, diagnosis, and treatment of common primary psychiatric causes of cutaneous disease // Journal of Dermatological Treatment. 2018. N 29(4). p. 418-427.

35. Lai J., Xu Z., Xu Y., Hu S. Reframing delusional infestation: perspectives on unresolved puzzles // Psychology Research and Behavior Management. 2018. N 11. p. $425-432$.

36. Lamahewa K., Buszewicz M., Walters K., Marston L., Nazareth I. Persistent unexplained physical symptoms: a prospective longitudinal cohort study in UK primary care // British Journal of General Practice. 2019. N 69(681). p. e246-e253

37. LaMotte R.H., Dong X., Ringkamp M. Sensory neurons and circuits mediating itch // Nature Reviews Neuroscience. 2014. N 15(1). p. $19-31$.

38. Levin E., Gieler U. Delusions of Parasitosis // Seminars in Cutaneous Medicine and Surgery. 2013. N 32. p.: 73-77.

39. Lloyd D.M., McGlone F.P., Yosipovitch G. Somatosensory pleasure circuit: from skin to brain and back // Experimental Dermatology. 2015. N 24(5). p. 321-324.

40. Magin P.J., Pond C.D., Smith W.T., Watson A.B., Goode S.M. Correlation and agreement of self-assessed and objective skin disease severity in a cross-sectional study of patients with acne, psoriasis, and atopic eczema // International Journal of Dermatology. 2011. N 50. p. 1486-1490.

41. Marcus D.K., Gurley J.R., Marchi M.M., Bauer C. Cognitive and perceptual variables in hypochondriasis and health anxiety: a systematic review // Clinical Psychology Review. 2007. N 27(2). p. 127-139.

42. McAndrew L.M., Friedlander M.L., Phillips L.A., Santos S., Helmer D.A. Concordance of illness perceptions: The key to improving care of medically unexplained symptoms // Journal of Psychosomatic Research. 2018. N 111. p. 140-142.

43. Misery L., Dutray S., Chastaing M., Schollhammer M., Consoli S.G., Consoli S.M. Psychogenic itch // Translational Psychiatry. 2018. N 8. p. 52. URL: https://www. ncbi.nlm.nih.gov/pmc/articles/PMC5830411/ (дата обращения: 20.08.2020).

44. Moriarty N., Alam M., Kalus A., O'Connor K. Current Understanding and approach to delusional infestation // American Journal of Medicine. 2019. N 132(12). p.1401-1409.

45. Neng J.M.B., Weck F. Attribution of somatic symptoms in Hypochondriasis // Clinical Psychology and Psychotherapy. 2015. N 22 (2). p. $116-124$.

46. Oliveirra E.C.B., Fitzpatrick C.L., Kim H.S., Gulassa D.C.R., Amaral R.S., Cristiana N.M., Hayashiuchi A.Y., McGrath D.S., Tavaresa H. Obsessive-compulsive or addiction? Categorical diagnostic analysis of excoriation disorder compared to obsessive-compulsive disorder and gambling disorder // Psychiatry Research. 2019. N 281. p. 112518.

47. Perepelkina, 0., Romanov, D., Arina, G., Volel, B., Nikolaeva, V. Multisensory mechanisms of body perception in somatoform disorders // Journal of Psychosomatic Research. 2019. N 127. p. 1-8.

48. RiefW., Barsky A.J. Psychobiological perspectives on somatoform disorders // Psychoneuroendocrinology. 2005. N 30. p. 996-1002.

49. Riem M.M.E., Doedéeb E.N.E.M., Broekhuizen-Dijksman S.C., Beijer E. Attachment and medically unexplained somatic symptoms: The role of mentalization // Psychiatry Research. 2018. N 268. p. 108-113.

50. Salkovskis P., Warwick H. Making Sense of Hypochondriasis: a Cognitive Model of Health Anxiety. // Asmundson G.S.G., Taylor S., Cox B.J. (Eds). Health Anxiety: Clinical and Research Perspectives on Hypochondriasis and Related Conditions. Chichester: Willey, 2001. p. 46-64.

51. Schaefer, M., Egloff, B., \& Witthöft, M. Is interoceptive awareness really altered in somatoform disorders? Testing competing theories with two paradigms of heartbeat perception // Journal of Abnormal Psychology. 2012. N 121(3). p. 719-724. 
52. Schmidt M. Psychic skin: psychotic defences, borderline process and delusions // Journal of Analytical Psychology. 2012. N 57. p. 21-39.

53. Schuck K., Keijsers G.P.J., Rinck M. The effects of brief cognitive-behaviour therapy for pathological skin picking: A randomized comparison to wait-list control // Behaviour Research and Therapy. 2011. N 49. p. 11-17.

54. Smulevich A.B., Lvov A.N., Romanov D.V. Hypochondriasis Circumscripta: A Neglected Concept with Important Implications in Psychodermatology // Acta DermatoVenereologica. 2016. N 96(217). p. 64-68.

55. Ulnik J. Skin in psychoanalysis. London: Karnac Books, 2008. 302 p.

56. van Laarhoven A.I.M, Kraaimaat F.W., Wilder-Smith 0.H., Evers A.W.M. Role of Attentional Focus on Bodily Sensations in Sensitivity to Itch and Pain // Acta Dermato-Venereologica. 2010. N 90. p.: 46-51.

57. Verhoeven E.W.M., Klerk S., Kraaimaat F.W., Van de Kerkhof P. C. M., De Jong E. M. G. J., Evers A. W. M. Biopsychosocial mechanisms of chronic itch in patients with skin diseases: a review // Acta Dermato-Venereologica. 2008. N 88. p. 211-218.

58. Yosipovitch G. Assessment of Itch: More to be Learned and Improvements to be Made. // Journal of Investigative Dermatology. 2003. N 121(6). p. xiv-xv.

59. Zachariae R., Zachariae C.O.C., Lei U., Pedersen A.F. Affective and Sensory Dimensions of Pruritus Severity: Associations with Psychological Symptoms and Quality of Life in Psoriasis Patients // Acta Dermato-Venereologica. 2008. N 88. p.121-127.

60. Zhu T.H., Nakamura M., Farahnik B., Abrouk M., Reichenberg J., Bhutani T., Koo J. Obsessive-compulsive skin disorders: a novel classification based on degree of insight // Journal of Dermatological Treatment. 2017. N 28(4). p. 342-346.

() Ермушева Анастасия Алексеевна (aermusheva@gmail.com).

Журнал «Современная наука: актуальные проблемы теории и практики»

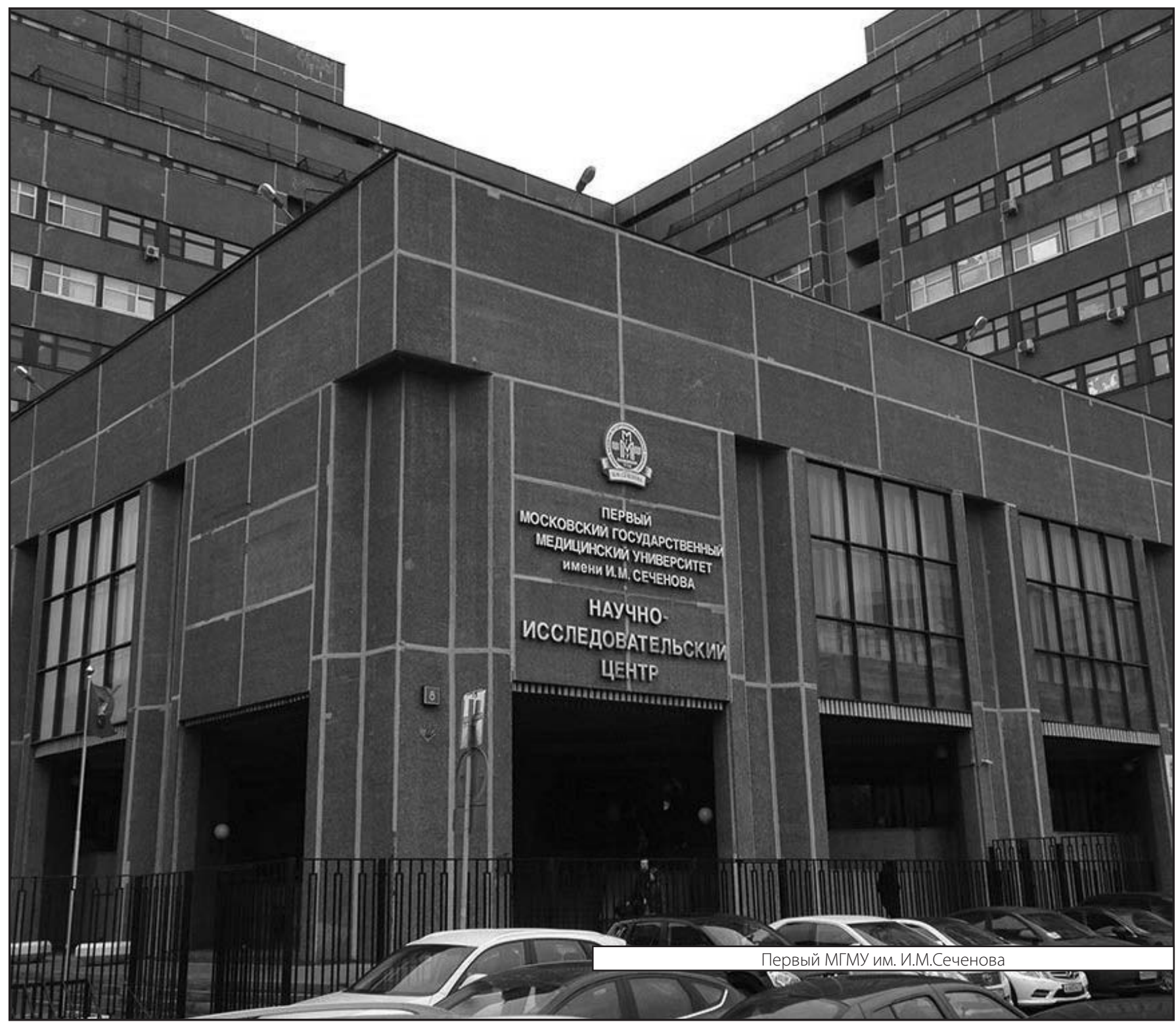

